\title{
Effect of 8 Weeks of Free-Weight and Machine-Based Strength Training on Strength and Power Performance
}

\author{
by \\ Klaus Wirth ${ }^{1}$, Michael Keiner ${ }^{2}$, Hagen Hartmann ${ }^{3}$, Andre Sander ${ }^{4}$; \\ Christoph $\mathrm{Mickel}^{3}$
}

\begin{abstract}
The aim of this study was to evaluate the effectiveness of free-weight and machine-based exercises to increase different strength and speed-strength variables. One hundred twenty male participants (age: $23.8 \pm 2.5$ years; body height: $181.0 \pm 6.8 \mathrm{~cm}$; body mass: $80.2 \pm 8.9 \mathrm{~kg}$ ) joined the study. The 2 experimental groups completed an 8 week periodized strength training program that included 2 training sessions per week. The exercises that were used in the strength training programs were the parallel barbell squat and the leg press. Before and after the training period, the 1repetition-maximum in the barbell squat and the leg press, the squat jump, the countermovement jump and unilateral isometric force (maximal isometric force and the rate of force development) were evaluated. To compare each group pre vs. post-intervention, analysis of variance with repeated measures and Scheffé post-hoc tests were used. The leg press group increased their 1-repetition-maximum significantly $(p<0.001)$, while in the squat group such variables as 1repetition-maximum, the squat jump and the countermovement jump increased significantly $(p<0.001)$. The maximal isometric force showed no statistically significant result for the repeated measures factor, while the rate of force development of the squat group even showed a statistically significant decrease. Differences between the 2 experimental groups were detected for the squat jump and the countermovement jump. In comparison with the leg press, the squat might be a better strength training exercise for the development of jump performance.
\end{abstract}

Key words: strength, power, diagnostics, squat, leg press.

\section{Introduction}

Strength training-induced increases in speed strength seem indisputable (Arabatzi et al., 2010; Christou et al., 2006). Several longitudinal investigations have found increases in squat jump (SJ) and countermovement jump (CMJ) performance after strength training interventions using different training exercises (Arabatzi et al., 2010; Christou et al., 2006). In this context, different effectiveness of free-weight compared to machine-based strength training is often discussed (Luebbers and Fry, 2011; Lyons et al.,

2010). In the comparison of the squat and leg press exercise, both exercises train nearly the same muscles of the lower extremities, but in some aspects they are different: the leg-press has less requirements concerning balancing the weight and therefore, less muscle activity contributes toward stabilization compared to the squat, however, the leg press allows more force to be applied in the linear path. Furthermore, the squat movement keeps the individual in an upright position, but the leg-press movement for

\footnotetext{
1 - Institute of Training and Sport, University of Applied Sciences Vienna Neustadt, Austria.

2 - Swimming Federation of the State Lower Saxony, Hannover, Germany.

3 - Institute of Sports Sciences, Department of Human Movement Science and Athletic Training, Johann Wolfgang Goethe-

University, Frankfurt, Germany.

4 - German Luge and Bobsled Federation, Berchtesgaden, Germany.
} 
example with a $45^{\circ}$ leg press is performed by the individual in a nearly supine position. Compared with the squat, the $45^{\circ}$ leg press spares the last $45^{\circ}$ extension motion in the hip and consequently the hip extensors are not trained in that range. The same issue can be applied to the seated leg press dependent on the inclination of the back cushion. The seated leg-press movement also has a horizontal/vertical movement pattern, whereas the squat requires almost a vertical press. Therefore, the specific adaptations have to be considered (Wilk et al., 1996).

However, differences in effectiveness always have to be discussed in the context of the desired training goal. It is conceivable that a strength training exercise that is optimal for gains in muscle hypertrophy may not be ideal for the development of speed-strength. Different conditions may be required for an exercise to meet these two training goals. For a gain in the muscle cross-sectional area, training with high loads is necessary to create the required high tension on the muscle fibers (Fry, 2004). To maximize performance in speed-strength, neurophysiological adjustments are required, at least in the short term (Zaras et al., 2014). This means that the greatest possible number of motor units must be recruited and addressed simultaneously with a high innervation frequency in a short time window (Duchateau and Hainaut, 2003). However, these timely constraints are of minor importance for hypertrophy effects. Continuous improvements in speed-strength are generated via concurrent strength and power exercises in a periodized fashion over years of resistance training (Baker and Newton, 2008), whereby maximum strength is regarded as the basic quality for high speed-strength performance (Pearson et al., 2002). Therefore, it may be expected that an increase in maximum strength alone is a sufficient training stimulus for the development of speed-strength in moderately trained subjects (Baker, 2001).

Additionally, the way of testing maximal force production is of importance. In the context of force measurements in competitive sports as well as in preventive and rehabilitative settings, isometric measurements are often carried out and recommended (Balyzer et al., 2015; Marcora and Miller, 2000). Balyzer et al. (2015) stated for testing under isometric conditions that a high number of subjects could be evaluated in a short period of time and that it was a safe method for testing maximum strength. However, previous studies had only reported statistically moderate to strong relationships between multi-joint isometric tests and dynamic movements such as vertical jumps (Kawamori et al., 2006), shot put and weighted throw performance (Stone et al., 2003). The problem for competitive sports is that muscles or muscle groups are mostly trained dynamically, but the level of strength and/or the rate of force development are analyzed under isometric conditions. Such an approach engenders the issue of strength increases achieved in training exercises, which cannot be confirmed in the diagnostic test procedure (Hartmann et al., 2009). Numerous documented specific adaptations to strength training, in this case primarily joint angle and contraction type-specific adaptations, may explain this problem (Knapik et al., 1983; PaddonJones et al., 2001; Seger and Thorstensson, 2005). Therefore, there is a risk of evaluating training and therapeutic successes in an incorrect way. Consequently, problems arise for the interpretation of standard data in health care or competitive sports, if they were determined through isometric measurements. From this perspective, it is also important to examine to what extent the relationship between isometric and dynamic testing is affected by the selected weight training exercises.

The aim of the study was to determine how the selection of the training exercise influenced speed-strength performance during an 8 week training intervention. In addition, we analyzed to what extent an increase of maximum strength affected an independent maximum strength variable. Furthermore, correlations between tested variables were calculated.

\section{Material and Methods}

\section{Participants}

One hundred twenty students (aged: 23.8 \pm 2.5 years; body height: $181.0 \pm 6.8 \mathrm{~cm}$; body mass: $80.2 \pm 8.9 \mathrm{~kg}$ ) of the Institute of Sports Science, Goethe University, Frankfurt, Germany, volunteered for the study. Each subject was informed of the experimental risks involved with the research. All subjects provided written informed consent to participate. The research design was approved by the institutional review 
board of the Institute of Sports Science, Goethe University, Frankfurt, Germany. The study was carried out with respect to the use of human subjects and according to the Declaration of Helsinki.

\section{Design}

The participants were divided into 3 groups. The $1^{\text {st }}$ group (SQ) completed an 8 week strength training protocol using the parallel barbell squat $\left(60-70^{\circ}\right.$ knee angle). The $2^{\text {nd }}$ group (LP) followed the same training protocol, but using the leg press $\left(45^{\circ}\right.$ leg press, $90^{\circ}$ knee angle). The $3^{\text {rd }}$ group $(\mathrm{CON})$ constituted a control group and did not perform resistance exercise during the experiment.

\section{Procedure}

The pretest was performed 3 days after a familiarization test, which included the same tests in the same order. The same tests were carried out again 3 days after the last training session. The tests were realized in the order described below after a standardized warm-up. The warm up consisted of 5 min of submaximal cycling on an ergometer and 2 to 3 sets of moderate loaded squats with 6 repetitions each.

First, the SJ (test-retest reliability $\mathrm{r}=0.87$; $p<0.05$ ), then the CMJ (test-retest reliability $\mathrm{r}=$ 0.94; $p<0.05$ ) performance were measured (5 trials each) using a contact mat (Refitronic, Schmitten, Germany) that operates like a switch. The system only provides information on whether the mat is loaded or not. Therefore, flight time is measured and further, the jump height calculated $\left(\frac{g t^{2}}{8} ; \mathrm{g}=\right.$ the gravitational acceleration $\left[9.81 \mathrm{~m} \cdot \mathrm{s}^{-2}\right]$ and $t=$ flight time). An error estimate can be found in Frick et al. (1991). The jumps were performed at a knee angle of $90^{\circ}$ with the hands fixed at the hips. In the SJ, subjects were asked to hold a static position of the $90^{\circ}$ knee angle for $2 \mathrm{~s}$, before they jumped (without momentum). In the $\mathrm{CMJ}$, subjects started in an upright position, then descended quickly to the $90^{\circ}$ knee angle before they jumped. The correct movement execution was controlled visually by the investigators. The subjects had a rest period of $2 \mathrm{~min}$ between jumps.

Isometric maximum force (MIF) was determined using a legwork machine (BAG, Wolf, Germany, test-retest reliability $r=0.90 ; p<0.01)$ in a seated position with a hip angle of $60^{\circ}$ and a knee angle of $120^{\circ}$. In addition, the rate of force development (RFD; test-retest reliability $r=0.77 ; p$ $<0.01)$ was determined from the force-time curves. Subjects had 3 attempts with a rest period of $5 \mathrm{~min}$ between attempts. The joint angles were controlled via a goniometer. Subjects were further encouraged to produce maximum strength as fast as possible and to continue the efforts for $3 \mathrm{~s}$. Therefore, we instructed them to build up maximal strength in an explosive fashion.

Preliminary studies for the BAG showed that the pressure load between the backrest (which is supported in the lower area of the back through several components made of steel) and the lumbar region of the back was considered very unpleasant, nearly painful, for the participants, when using both legs for testing. To avoid inhibitory influences (resulting from pain) on the development of speed-strength, a unilateral test was performed. The $120^{\circ}$ knee joint angle was chosen as it represents a favorable position for the development of explosive force production (Hemmling, 1994). Larger angles would lead to lower maximum force values (Hemmling, 1994). In addition, based on preliminary studies, subjects described knee joint angles of $90^{\circ}$ or less as unpleasant to painful.

Maximum dynamic strength was determined through the load of the one repetition maximum (1RM) in the squat or the leg press 15 min after determining MIF. The maximal load was determined in a series of 1RM. Determination of the 1RM was achieved within a maximum of 5 trials. Rest periods between attempts were at least $5 \mathrm{~min}$. The criterion for a successful attempt in the squat was a trial in which the upper part of the knee musculature was parallel to the floor at the turning point and the leg was completely extended in the upright position. Attempts failed when the subjects rounded their back, lost the bar, or where not able to flex the knees to the desired depth. In the leg press (Rowe \& Kopp, Oberursel, Germany), the load was lowered to a knee joint angle of $90^{\circ}$. Range of motion was monitored using a goniometer attached to a knee brace. Subjects wore the knee brace at their right knee. At the starting position (with both legs on the plate) a trigger was set. The test-retest reliability coefficient for the leg press was $\mathrm{r}=0.96(p<0.05)$ and $\mathrm{r}=0.92$ for the squat $(p<0.05)$.

The training groups performed 5 sets of their 8-10 repetitions maximum (RM) during the 
first 3 weeks. Thereafter, training groups performed 5 sets of $6-8 \mathrm{RM}$ in the 4 th to 6 th week, and 5 sets of 4-6 RM in the 7th and 8 th weeks. Subjects were always allowed $5 \mathrm{~min}$ of rest between sets. The difference between the training groups was the selected exercise only (squat vs. leg press). Generally, bouncing the bar in the eccentric-concentric transition phase was not allowed. The subjects performed each set to momentary muscular failure in the last 2 repetitions of the targeted repetitions scheme (forced reps). The researchers provided spotting and strong verbal encouragement. If necessary, resistance was adapted by $2.5-10 \mathrm{~kg}$ for the next set or next training session for the subject to stay in the particular repetition scheme.

\section{Statistical Analysis}

Data were analyzed with SPSS 17.0. It was checked for normality using the KolmogorovSmirnov test. For all group comparisons and comparisons between pre- and post-intervention results, 2-factorial analyses of variance were performed using a repeated measures model. The Mauchly sphericity test was performed prior to ANOVAs. If sphericity was calculated as statistically significant, the Greenhouse-Geisser correction was used. When statistically significant $F$ values were returned, the Scheffé's test was used for further post hoc analyses. Since different tests of maximum dynamic strength (squat and leg press) cannot be directly compared, the variation of maximum strength measures over the study period was analyzed using the t-test for paired samples. The correlation between the tested variables was calculated by the Pearson's product-moment coefficient. The level of significance for all statistical tests was set at $p<$ 0.05 . The relationships were classified as follows: $0=$ no correlation, $0<|\mathrm{r}|<0.2$ = very weak correlation, $0.2 \leq|\mathrm{r}|<0.4=$ weak correlation, 0.4 $\leq|\mathrm{r}|<0.6=$ moderate correlation, $0.6 \leq|\mathrm{r}|<0.8=$ strong correlation, $0.8 \leq|\mathrm{r}|<1.0=$ very strong correlation, 1 = perfect correlation.

\section{Results}

The Kolmogorov-Smirnov test showed the data were normally distributed for all test variables. Furthermore, the homogeneity of variance between groups was confirmed using the Levene test for all variables. The anthropometric data are presented in Table 1.
The ANOVA with repeated measures showed, for both the maximum isometric force of the left leg (MIF L, $p>0.05$ ) and the maximum isometric force of the right leg (MIF R, $p>0.05$ ), no statistically significant result for the repeated measures factor. However, for both variables, a statistically significant result in the comparison of groups was found (MIF L: $p \leq 0.001$; MIF-R: $p \leq$ $0.001)$. In both cases, statistically significant group differences between the $\mathrm{CON}$ and SQ groups (MIF-L: $p \leq 0.001$; MIF-R: $p \leq 0.001$ ) and between the CON and LP groups (MIF-L: $p \leq 0.001$; MIF-R: $p \leq 0.01)$ were calculated. The results are presented in Table 2.

The ANOVA with repeated measures showed, for both the RFD of the left (RFD-L, $p \leq$ 0.05 ) and the right leg (RFD-R, $p \leq 0.001$ ), a statistically significant result for the repeated measures factor. The results of the RFD are presented in Table 3. However, for neither variable a statistically significant result in the comparison of groups (RFD-L: $p>0.05$; RFD-R: $p>$ 0.05) was found. For both, the left and the right leg, a statistically significant decrease of the RFD was observed in the control group. In addition, the results of the SQ group showed a statistically significant decrease in the RFD.

The ANOVA with repeated measures showed, for both the squat jump (SJ, $p \leq 0.001$ ) and the countermovement jump (CMJ, $p \leq 0.001$ ), a statistically significant result for the repeated measures factor. For both variables, a statistically significant result for the group comparison was detected (SJ: $p \leq 0.001$; CMJ: $p \leq 0.001$ ). In both cases, statistically significant group differences were found for all 3 group comparisons ( $p \leq$ $0.001)$. The changes in performance in the SJ and the CMJ from pre- to post-intervention showed a statistically significant improvement in both variables in the SQ group and a statistically significant decrease in performance in the SJ in the CON group. The results are presented in Table 4.

The t-test for paired samples showed a statistically significant increase in maximum dynamic strength for both groups (SQ: $p \leq 0.001$; LP: $p \leq 0.001)$. For the MIF-LR variables, the ANOVA with repeated measures showed a statistically significant result for the group comparison ( $p \leq 0.001)$, but no statistically significant result for the repeated measures factor $(p>0.05)$. Both training groups $(\mathrm{SQ}, \mathrm{LP})$ differed 
significantly from the control group (CON) in their performance increases $(p \leq 0.001)$. The results are presented in Table 5.

The correlations are presented in Tables 6 and 7. The correlations in the SQ group were mostly statistically significant. Only the correlations between the RFD-L and the SJ as well as between the RFD-L and the CMJ were not statistically significant.

In the LP group all correlation coefficients reached the level of significance.

\section{Table 1}

Anthropometric data of the participants

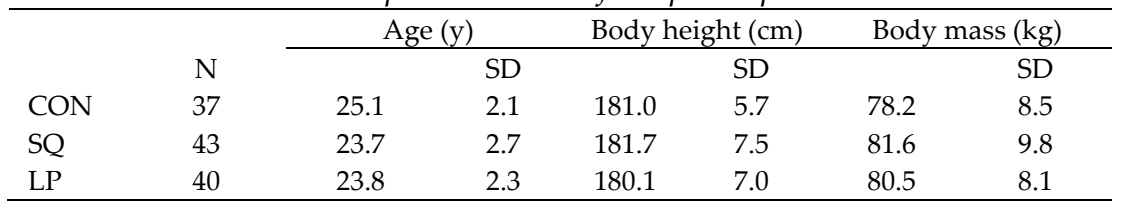

$=$ mean values; $S D=$ standard deviations; $y=$ years $; \mathrm{cm}=$ centimeters; $\mathrm{kg}=$ kilogram;

$C O N=$ control group; $S Q=$ squat group; $L P=$ leg press group

\section{Table 2}

Means, standard deviations and changes in maximum isometric force (N) from pre-to post-test

\begin{tabular}{|c|c|c|c|c|c|c|c|c|c|c|}
\hline & \multicolumn{5}{|c|}{ MIF-L } & \multicolumn{5}{|c|}{ MIF-R } \\
\hline & \multicolumn{2}{|c|}{ T1 } & \multicolumn{2}{|c|}{$\mathrm{T} 2$} & & \multicolumn{2}{|c|}{ T1 } & \multicolumn{2}{|c|}{$\mathrm{T} 2$} & \multirow[b]{2}{*}{$\%$} \\
\hline & & SD & & $\mathrm{SD}$ & $\%$ & & SD & & SD & \\
\hline $\mathrm{CON}$ & 2127 & 565 & 1979 & 471 & -5.7 & 2147 & 510 & 2030 & 484 & -4.9 \\
\hline SQ & 2253 & 478 & 2389 & 511 & 6.7 & 2310 & 463 & 2408 & 536 & 4.4 \\
\hline$\widehat{L P}$ & 2191 & 498 & 2250 & 473 & 3.1 & 2230 & 479 & 2319 & 521 & 4.5 \\
\hline
\end{tabular}

$=$ mean values; $S D=$ standard deviations; $M I F-L=$ maximum isometric force of the left leg;

$M I F-R=$ maximum isometric force of the right leg; $\%=$ percent; $T 1=$ pretest;

$\mathrm{T} 2$ = post-test $N=$ newtons; $C O N=$ control group; $S Q=$ squat group; $L P=$ leg press group

Table 3

Means, standard deviations and changes in the rate of force development $(\mathrm{N} / \mathrm{ms})$ from pre- to post-testing

\begin{tabular}{lcccccccccc}
\hline & \multicolumn{9}{c}{ RFD-L } & \multicolumn{3}{c}{ RFD-R } \\
\cline { 2 - 11 } & \multicolumn{3}{c}{ T1 } & \multicolumn{3}{c}{ T2 } & \multicolumn{3}{c}{ T1 } & \multicolumn{3}{c}{ T2 } \\
nyN & & SD & & SD & $\%$ & & SD & & SD & $\%$ \\
SQ & 11.2 & 3.0 & 10.2 & 2.9 & $-7.9^{*}$ & 11.5 & 2.5 & 10.8 & 2.7 & $-6.2^{*}$ \\
LP & 12.6 & 2.6 & 12.4 & 3.1 & -0.4 & 13.3 & 2.6 & 12.4 & 2.3 & $-5.8^{*}$ \\
\hline
\end{tabular}

$=$ mean values; $S D=$ standard deviations; $R F D-L=$ rate of force development of the left leg;

$R F D-R=$ rate of force development of the right leg; \% = percent; T1 = pretest;

$T 2$ = posttest; $N=$ newtons; ${ }^{*}=$ significantly $(p<0.05)$ different from the pretest within a group; $C O N=$ control group; $S Q=$ squat group; $L P=$ leg press group 


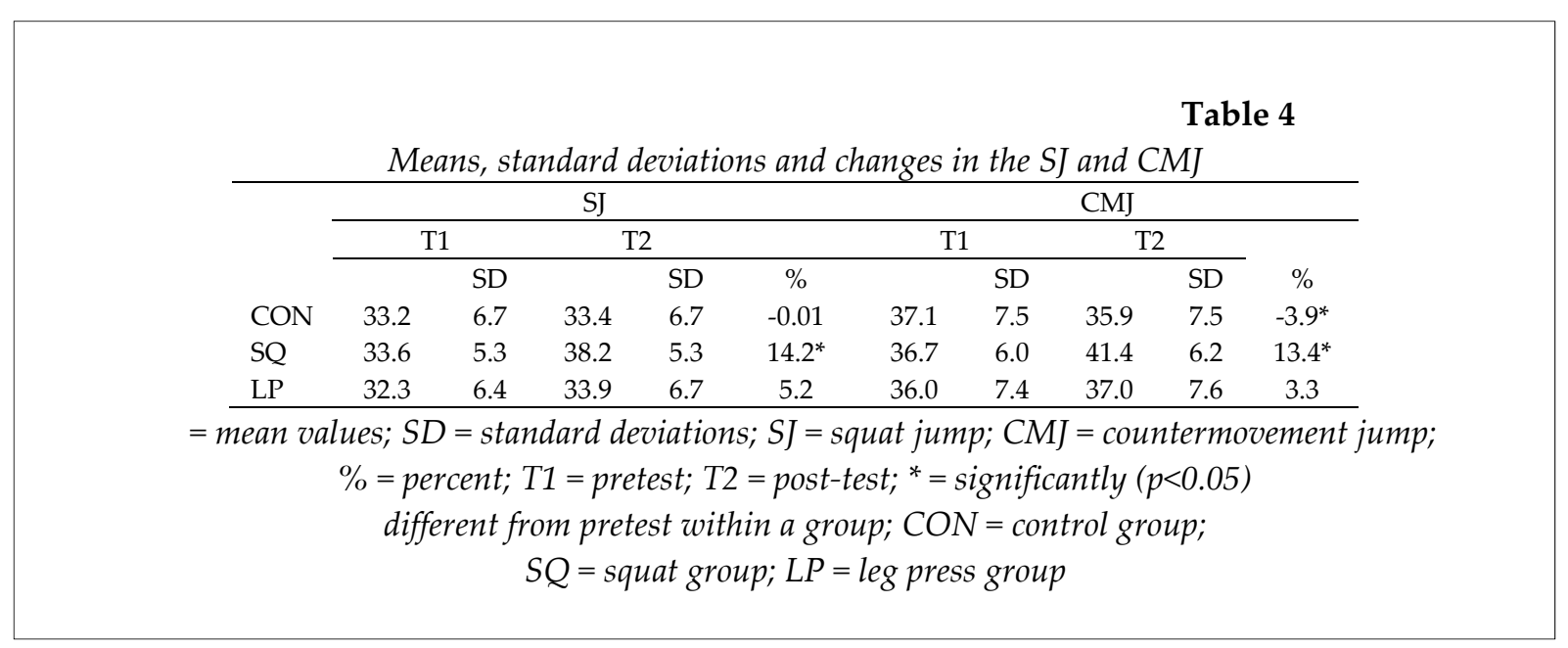

\begin{tabular}{|c|c|c|c|c|c|c|c|c|c|c|}
\hline \multicolumn{11}{|c|}{$\begin{array}{l}\text { Table } 5 \\
\text { Means, standard deviations and changes in dynamic maximum strength (squat/leg } \\
\text { press; }[\mathrm{kg}]) \text { and maximum isometric strength (sum of the } 2 \text { values } \\
\text { from the unilateral testing }[\mathrm{N}])\end{array}$} \\
\hline & \multicolumn{5}{|c|}{$1 \mathrm{RM}$} & \multicolumn{5}{|c|}{ MIF-LR } \\
\hline \multirow{4}{*}{$\mathrm{CON}$} & \multicolumn{2}{|c|}{$\mathrm{T} 1$} & \multicolumn{2}{|c|}{$\mathrm{T} 2$} & \multicolumn{3}{|c|}{$\mathrm{T} 1$} & \multicolumn{2}{|c|}{$\mathrm{T} 2$} & \multirow[b]{2}{*}{$\%$} \\
\hline & & SD & & SD & $\%$ & & SD & & SD & \\
\hline & 75.6 & 23.9 & 75.9 & 21.0 & 1.7 & 4236 & 1003 & 3984 & 905 & -5.2 \\
\hline & 220.7 & 88.1 & 226.9 & 64.7 & 7.7 & & & & & \\
\hline SQ & 97.1 & 29.0 & 118.0 & 29.4 & $23.9^{*}$ & 4563 & 904 & 4797 & 1010 & 5.4 \\
\hline LP & 230.3 & 57.4 & 296.8 & 68.3 & $30.5^{*}$ & 4435 & 933 & 4576 & 959 & 3.7 \\
\hline \multicolumn{11}{|c|}{$\begin{array}{c}=\text { mean values; } S D=\text { standard deviations; } S J=\text { squat jump; } C M J=\text { countermovement jump; } \\
\%=\text { percent } ; T 1=\text { pretest } ; T 2=\text { post-test } ;{ }^{*}=\text { significantly }(p<0.05) \text { different } \\
\text { from the pretest within a group; } C O N=\text { control group; } S Q=\text { squat group; } \\
L P=\text { leg press group; } 1 R M=\text { one-repetition maximum; } \\
M I F-L R=\text { maximum isometric force of the left and right leg. }\end{array}$} \\
\hline
\end{tabular}

\begin{tabular}{|c|c|c|c|c|c|c|c|}
\hline & & & & & \multicolumn{3}{|c|}{ Table 6} \\
\hline \multicolumn{8}{|c|}{ Correlations of strength and speed-strength variables in the SQ group $(n=43)$} \\
\hline & MIF-R & RFD-L & RFD-R & SJ & $\mathrm{CMJ}$ & MIF-LR & $1 \mathrm{RM}$ \\
\hline MIF-L & $0.86^{*}$ & $0.51^{*}$ & $0.47^{*}$ & $0.45^{*}$ & $0.40^{*}$ & $0.96^{*}$ & $0.70^{*}$ \\
\hline MIF-R & 1 & $0.46^{*}$ & $0.57^{*}$ & $0.51^{*}$ & $0.48^{*}$ & $0.97^{*}$ & $0.69^{*}$ \\
\hline RFD-L & & 1 & $0.70^{*}$ & 0.20 & 0.22 & $0.50^{*}$ & $0.54^{*}$ \\
\hline RFD-R & & & 1 & $0.46^{*}$ & $0.50^{*}$ & $0.54^{*}$ & $0.52^{*}$ \\
\hline SJ & & & & 1 & $0.96^{*}$ & $0.50^{*}$ & $0.72^{*}$ \\
\hline $\mathrm{CMJ}$ & & & & & 1 & $0.50^{*}$ & $0.64^{*}$ \\
\hline MIF-LR & & & & & & 1 & $0.72^{*}$ \\
\hline \multicolumn{8}{|c|}{$\begin{array}{l}M I F-L=\text { maximum isometric force of the left leg; } M I F-R=\text { maximum isometric } \\
\text { force of the right leg; } M I F-L R=\text { maximum isometric force of the left and right leg; } \\
\text { RFD- } L=\text { rate of force development of the left leg; } \\
R F D-R=\text { rate of force development of the right leg; } \\
\text { SJ = squat jump; } C M J=\text { countermovement jump; }{ }^{*}=\text { significant }(p<0.05)\end{array}$} \\
\hline
\end{tabular}


Table 7

Correlations of strength and speed-strength variables of the LP group $(n=40)$

\begin{tabular}{lccccccc}
\hline & MIF-R & RFD-L & RFD-R & SJ & CMJ & MIF-LR & 1RM \\
\cline { 2 - 8 } MIF-L & $0.85^{*}$ & $0.60^{*}$ & $0.58^{*}$ & $0.48^{*}$ & $0.49^{*}$ & $0.96^{*}$ & $0.54^{*}$ \\
MIF-R & 1 & $0.68^{*}$ & $0.76^{*}$ & $0.56^{*}$ & $0.57^{*}$ & $0.97^{*}$ & $0.67^{*}$ \\
RFD-L & & 1 & $0.87^{*}$ & $0.51^{*}$ & $0.53^{*}$ & $0.67^{*}$ & $0.54^{*}$ \\
RFD-R & & & 1 & $0.56^{*}$ & $0.56^{*}$ & $0.70^{*}$ & $0.57^{*}$ \\
SJ & & & & 1 & $0.97^{*}$ & $0.56^{*}$ & $0.66^{*}$ \\
CMJ & & & & & 1 & $0.57^{*}$ & $0.68^{*}$ \\
MIF-LR & & & & & & 1 & $0.64^{*}$ \\
\hline
\end{tabular}

$M I F-L=$ maximum isometric force of left leg; MIF-R = maximum isometric force of right leg;

$M I F-L R=$ maximum isometric force of left and right leg;

$R F D-L=$ rate of force development of left leg; RFD- $R$ = rate of force development of right leg;

$S J=$ squat jump; $C M J=$ countermovement jump; ${ }^{*}=$ significant $(p<0.05)$

\section{Discussion}

The main findings of this study include: first, the comparison of the squat and the leg press shows the squat to be a more effective exercise for increasing jump performance in short-term strength training; second, collecting strength/force data, the procedure should be carefully considered based on the desired test variables.

Statistically significant changes $(p \leq 0.05)$ in maximum dynamic strength were found for both training groups, but the isometric maximum force did not improve significantly. This observation may be explained in two different ways: numerous investigations have shown that increases in performance tests are greatest if training was performed in the same contraction type that is performance limiting (Farting and Chillibeck, 2003; Paddon-Jones et al., 2001). This is due to divergent innervation strategies used by the central nervous system (CNS; Kinugasa et al., 2005; Komi et al., 2000). For both training groups concentric phases of the exercises were performance-limiting. Since most of the increases in maximum strength observed are likely to be primarily due to neural adaptations (Aargaard et al., 2002; Moritani and DeVries, 1979), it is further likely that the enhanced activation of the lower extremities could not be retrieved in the isometric testing conditions.

The measurement of the $1 R M$ was performed bilateral compared to unilateral measurements of the isometric force production. As studies on the bilateral deficit show (Häkkinen et al., 1996; Howard and Enoka, 1991), the rate of performance change is influenced by the test conditions. However, the accumulated benefits of unilateral tests are higher than for bilateral tests. Therefore, the MIF LR should have shown an especially good performance increase, but this was not the case. Another difficulty arises from the fact that an isometric measurement has to be angle specific by definition. As maximum isometric force was determined at the $120^{\circ}$ knee joint angle (for previously described reasons), the highest activity of the central nervous system is found at about $60-70^{\circ}$ in the squat and at $90^{\circ}$ in the leg-press. Thus, it can be assumed that the angle-specific adjustments played an important role in our observations (Knapik et al., 1983). This demonstrates a problem in diagnostic practice as data of strength variables are often assessed in isometric conditions. Especially in short-term studies of only a couple of weeks intervention period, one could assume isometric maximum testing not to be sensitive enough to reveal increases in strength performance through dynamic exercises.

Considering the results of the collected speed-strength variables, a similar problem might occur. None of the groups showed statistically significant improvements in the RFD. On the 
contrary, the LP group even presented a significantly decreased RFD of the right leg. Based on the improvements of the other variables in this group it cannot be attributed to fatigue effects. Whether subjects were less motivated performing the isometric tests compared to dynamic measurements cannot be answered. Furthermore, no significant changes in the SJ were observed, whereas a significant decrease in the CMJ performance occurred. Given that both variables were highly correlated $(\mathrm{r}=0.97, \mathrm{p} \leq 0.01)$, this is surprising, especially since the CMJ is not such a complex motor task that its execution might have negatively affected the results.

However, even more surprising are the differences found in the development of jump performance of both training groups. The SQ group presented highly significant increases in the SJ $(14.2 \%)$ and CMJ (13.4\%), whereas no statistically significant changes were found for the LP group (SJ increased by $5.2 \%$ and CMJ by $3.3 \%$ ). As the differences between both training groups were also statistically significant, it can be concluded that training using the barbell squat is superior to the leg-press (concerning increases in jump performance). Probably, the greater benefit of the barbell squat is that the body position corresponds better with the tested jumps. The similarities of the squat and the SJ / CMJ are likely to facilitate the transfer of performance increases. Therefore, it seems to be easier for the CNS to transfer a high level of activation between these three motor tasks compared to the leg-press. Aside from this, it was still surprising that changes of the LP group did not reach significance as the maximum dynamic strength gains were high. Therefore, it was expected to find at least some transfer of the enhanced ability to activate the muscles in the SJ and CMJ.

In the first step, the analysis of correlations was performed separately for both training groups. That is why we were able to determine if the correlations between maximum strength and the other variables were influenced by the choice of strength training exercise. In the second step, the correlations were calculated for the entire sample. The relationship between maximum dynamic strength and isometric force (MIF LR) for the entire sample was $r=0.64$ ( $p \leq$ 0.01 ) and therefore, similar to the correlation between the maximal leg-press performance and isometric measures. The SQ group alone had $\mathrm{r}=$ $0.72(\mathrm{p} \leq 0.01)$ and the combination of SQ and Con groups showed $r=76(p \leq 0.01)$. The correlations for the two maximal strength measures were between $r=0.64$ to $r=0.76$ for the different sample groups and thus, remarkably low. For comparison, Schmidtbleicher (2003) reported correlation coefficients of $r>0.85$ up to $r>0.90$ for athletes. Once again, different hip and knee angles, contraction types and uni- vs. bilateral testing seem to exhibit a great influence on the results and therefore, suppress the expected high correlation coefficients. We found even lower correlation coefficients between the RFD and 1RM (range from $\mathrm{r}=0.52$ to $\mathrm{r}=0.61 ; \mathrm{p} \leq 0.01$ ) and correlation coefficients between 0.51 and 0.76 ( $\mathrm{p} \leq$ 0.01 ) for the comparison of the RFD with the isometric measures. Correlation coefficients around $\mathrm{r}=0.70$ for the relationship between maximal strength and the RFD confirm the basic assumption that speed-strength is highly dependent of the maximum force production capacity. The correlation coefficients for jump performance and the RFD are slightly lower as the aforementioned factors affect this relationship (between 0.46 and $0.56 ; \mathrm{p} \leq 0.01$ ). The values for the relationship between the RFD-L and SJ and CMJ for the LP group, $r=0.20(p>0.05)$ and $r=$ 0.22 ( $p>0.05)$, respectively, are difficult to explain.

The influence of maximum dynamic strength on jump performance should be considered as moderate to high ( $\mathrm{r}=0.64$ to 0.79 , $\mathrm{p}$ $\leq 0.01$ ), depending on the test conditions. This is in accordance with our expectations. The type of testing is therefore not important in maximum dynamic strength of the lower extremities. Correlations to maximum isometric measures are significantly lower (between $r=0.40$ and $r=0.57$; $p \leq 0.01$ ). Furthermore, changes in the contraction type, different joint angles and possibly uni- vs. bilateral testing perturb the correlations.

\section{Practical Implications}

Based on the present findings there are two implications for coaches and athletes: first, our data suggest a large influence of selection of the training exercise on short term performance improvement. The squat was shown to be significantly more effective compared to the legpress. Therefore, in the pre-competitive period the 
barbell squat should be preferred, owing to better transfer effects on jump performance. Second, when collecting strength data, the procedure should be carefully considered. The data presented here indicate the problems arising when measurements based on motor tasks with dynamic execution (when the joint angle varies over the range of motion) are compared to values collected with other contraction types and at a specified joint angle (isometric measurement, often used in therapy and comprehensive sport).

\section{References}

Aagaard P, Simonsen EB, Andersen JL, Magnusson SP, Dyhre-Poulsen P. Increased rate of force development and neuronal drive of human skeletal muscle following resistance training. $J$ Appl Physiol, 2002; 93: 1318-1326

Arabatzi F, Kellis E, De Villareal E. Vertical jump biomechanics after plyometric, weight lifting, and combined (weightlifting + plyometric) training. I Strength Cond Res, 2010; 24: 2440-2448

Baker D. Comparison of maximum upper body strength and power between professionals and college-aged rugby league players. J Strength Cond Res, 2001; 15: 30-35

Baker DG, Newton RU. Observation of 4-year adaptations in lower body maximal strength and power output in professional rugby league players. J Aust Strenth Cond, 2008; 18: 3-10

Bazyler CD, Beckham GK, Sato K. The use of the isometric squat as a measure of strength and explosiveness. J Strength Cond Res, 2015; 29: 1386-1392

Christou M, Smilios I, Sotiropoulos K, Volaklis K, Pilianidis T, Tokmakidis SP. Effects of resistance training on the physical capacities of adolescent soccer players. J Strength Cond Res, 2006; 20: 783-791

Farthing JP, Chilibeck PD. The effects of eccentric and concentric training at different velocities on muscle hypertrophy. Eur J Appl Physiol, 2003; 89: 578-586

Fry AC. The role of resistance exercise intensity on muscle fibre adaptations. Sports Med, 2004; 34: 663-679

Frick U, Schmidtbleicher D, Wörn C. Comparison of biomechanical measuring methods for determining the jump height in vertical jumps (transl.). Leistungssport, 1991; 21: 48-53

Hartmann H, Bob A, Wirth K, Schmidtbleicher D. Effects of different periodization models on rate of force development and power ability of the upper extremity. J Strength Cond Res, 2009; 23: 1921-1932

Duchateau J, Hainaut K. Mechanisms of Muscle and Motor Unit Adaptation to Explosive Power Training. In Komi PV: Strength and Power in Sport. Oxford: Blackwell Science, 316 - 330; 2003

Hemmling G. Adaptations of the neuromuscular system to a newly developed method of training. Colone: Federal Institute for Sport Science; 1994

Howard JD, Enoka RM. Maximum bilateral contractions are modified by neurally mediated interlimb effects. J Appl Physiol, 1991; 70: 306-316

Häkkinen K, Kallinen M, Linnamo V, Pastinen UM, Newton RU, Kraemer WJ. Neuromuscular adaptations during bilateral versus unilateral strength training in middle-aged and elderly men and women. Acta Physiol Scand, 1996; 158: 77-88

Kawamori N, Rossi SJ, Justice BD, Haff EE, Pistilli EE, O'Bryant HS, Stone MH, and Haff GG. Peak force and rate of force development during isometric and dynamic mid-thigh clean pulls performed at various intensities. J Strength Cond Res, 2006; 20: 483-491

Kinugasa R, Kawakami Y, Fukunaga T. Three-dimensional activation properties of triceps surae muscles during concentric and eccentric contractions using muscle functional MRI. Med Sci Sports Exerc, 2005; 3: $440-441$

Knapik JJ, Mawdsley RH, Ramos MU. Angular specificity and test mode specificity of isometric and isokinetic strength training. J Orthop Sports Phys Ther, 1983; 2: 58-65 
Komi PV, Linnamo V, Silventoinen P, Sillanpää M. Force and EMG power spectrum during eccentric and concentric actions. Med Sci Sports Exerc, 2000; 32: 1757-1762

Linnamo V, Strojnik V, Komi PV. EMG power spectrum und features of the superimposed M-wave during voluntary eccentric and concentric actions at different activation levels. Eur J Appl Physiol, 2002; 86: 534-540

Luebbers PE, Fry AC. The Kansas squat test protocol comparision: Smith machine vs free-weights. J Strength Cond Res, 2011; 25: S670S68

Lyons TS, McLester JR, Arnett SW, Thoma MJ. Specificity of training modalities on upper-body one repetition maximum performance: free weights vs. hammer strength equipment. J Strength Cond Res, 2010; 24: 2984-2988

Marcora S, Miller MK. The effect of knee angle on the external validity of isometric measures of lower body neuromuscular function. J Sports Sci, 2000; 18: 313-319

Moritani T, DeVries HA. Neural factors versus hyperthrophy in the time course of muscle strength gain. $A m$ J Med, 1979; 58: 115-130

Paddon-Jones D, Leveritt M, Lonergan A, Abernethy P. Adaptation to chronic eccentric exercise in humans: the influence of contraction velocity. Eur J Appl Physiol, 2001; 85: 466-471

Pearson SJ, Young A, Macaluso A, Devito G, Nimmo MA, Cobbold M, Harridge SDR. Muscle function in elite master weightlifters. Med Sci Sports Exerc, 2002; 34: 1199-1206

Rabita G, Pérot C, Lensel-Corbeil G. Differential effect of knee extension isometric training on the different muscles of the quadriceps femoris in humans. Eur J Appl Physiol, 2000; 83: 531-538

Schmidtbleicher D. Strength: structure, components, adaptation symptoms, training methods and periodization. In: Fritsch W, ed. Rowing - learn, explore, research. Gießen: Sport Media, 15-40; 2003

Seger JY, Thorstensson A. Effects of eccentric versus concentric training on thigh muscle strength and EMG. Int J Sports Med, 2005; 26: 45-52

Wilk KE, Escamilla RF, Felig GA, Barrentine SW, Andrews JR, Boyd ML. A comparison of tibiofemoral joint forces and electromyographic activity during open and closed kinetic chain exercises. Am J Sports Med, 1996; 24: 518-527

Zaras ND, Stasinaki AN, Krase AA, Methenitis SK, Karampatsos GP, Georgiadis GV, Spengos KM, Terzis GD. Effects of tapering with light vs. heavy loads on track and field throwing performance. J Strength Cond Res, 2014; 28: 3484-95

\section{Corresponding author:}

\section{Michael Keiner}

Swimming Federation of the State Lower Saxony, Hannover, Germany

Ferdinand-Wilhelm-Fricke-Weg 10

30169 Hannover

Phone: +491632784652

E-Mail: Michaelkeiner@gmx.de 